\title{
Faringo-íleo-colo anastomosis con suplemento vascular microquirúrgico en reconstrucción del tracto digestivo superior
}

\author{
Italo Braghetto M. ${ }^{1}$, Manuel Figueroa-Giralt ${ }^{1}$, Belén Sanhueza P. ${ }^{1}$, Enrique Lanzarini S. ${ }^{1}$, \\ Sergio Sepúlveda P. ${ }^{1}$, Cristian Erazo C. ${ }^{1}$ y Rolando González C. ${ }^{1}$
}

Departamento de Cirugía Hospital Clínico Universidad de Chile, Santiago, Chile.

Recibido el 4 de agosto de 2017, aceptado para publicación el 9 de noviembre de 2017

Correspondencia a: Dr. Italo Braghetto M. ibraghetto@hcuch.cl

\section{Pharyngo-ileo-colo-anastomosis with micro-vascular blood supply augmentation for surgical reconstruction of the upper digestive tract}

Introduction: Patients submitted to total esophagectomy and gastrectomy with complete closure of pharinx due to necrosis after caustic ingestion are a challenging surgical setting for reconstruction of upper digestive transit. Objective: The objective of this paper is to present a clinical case and surgical technique for reconstruction of the upper digestive tract after total esophagectomy and gastrectomy. Method: Reconstruction of digestive transit was reestablished by means of a pharyngo-ileo-colonic interposition with microsurgical arterial and venous anastomosis for augmentation of blood supply. Results: There were not major postoperative complications and at long term follow-up, normal oral nutrition and quality of life improvement was observed. Conclusion: This is a surgical procedure for treatment of patients with pharyngeal strictures without any possibility to indicate other less complex procedures.

Key words: esophagectomy; esophageal replacement; colon interposition; microsurgical anastomosis.

\section{Resumen}

Introducción: Los pacientes sometidos a desconexión total con cierre al nivel del seno piriforme debido a necrosis completa del esófago y estómago después de la ingestión cáustica representan un desafío quirúrgico para restablecer la ingestión oral y la calidad de vida. Objetivo: El objetivo de este trabajo es presentar la experiencia con un caso clínico con necrosis total de esófago y estómago posingestión de cáuticos por lo que fue inicialmente sometido a esofagectomía y gastrectomía total. Método: La reconstrucción del tracto digestivo superior se efectuó mediante una faringo-íleo-colo anastomosis, con suplemento de irrigación sanguínea arterial y drenaje venoso mediante técnica de anastomosis microquirúrgica. Resultados: No se observaron complicaciones postoperatorias mayores y en el resultado a largo plazo se logra alimentación oral normal con una recuperación nutricional adecuada y buena calidad de vida. Conclusión: Esta es un procedimiento a plantear en pacientes con estenosis faríngea sin posibilidad de reemplazo esofágico con procedimientos menos complejos.

Palabras clave: esofaguectomía; reemplazo esofágico; interposición de colon; anastomosis microquirúrgica.

\section{Introducción}

En la actualidad las opciones quirúrgicas para la reconstitución primaria de tránsito esofágico incluyen técnicas como ascenso de estómago, colon y raramente el yeyuno. Existen situaciones clínicas excepcionales en las cuales el tránsito desde la faringe hacia el tracto digestivo distal se ve imposi- bilitado pues no se dispone de esófago y estómago utilizable ya sea por injuria previa (ingestión de cáusticos), resección previa del esófago y estómago o por necrosis del sustituto. En estas condiciones, la reconstrucción del tránsito digestivo superior en ausencia de esófago funcional y/o anatómico es un desafío quirúrgico y las alternativas terapéuticas incluyen grandes cirugías con una morbimortalidad 
considerable $\mathrm{e}^{1-3}$. Después de cirugías previas fallidas con estenosis y desconexión del tracto digestivo, la técnica de reconstitución del tracto digestivo alto depende de qué órgano se dispone para ser utilizado. Una alternativa más conservadora es la reutilización del estómago ascendido y reanastomosis esófago-gástrica, pero esto no siempre es fácil o posible. Por lo que frecuentemente debemos efectuar una cirugía utilizando interposición de colon. La morbilidad quirúrgica asociada a este tipo de intervenciones puede ser entre $50 \%$ y $70 \%{ }^{4-7}$.

Una dificultad extra se presenta cuando no existe esófago cervical disponible para la reconstrucción y existe estenosis total a nivel faríngeo como ocurre en los pacientes con ingestión de cáusticos, lo que hace plantear procedimientos aún más complejos para poder efectuar anastomosis segura sin isquemia a nivel faríngeo. El objetivo de este trabajo, es presentar un caso clínico y la técnica quirúrgica de reconstrucción del tránsito esofágico, por medio de una anastomosis faringo-íleo-cólica con suplemento vascular microquirúrgico.

\section{Material y Método}

\section{Caso clínico}

Se presenta la experiencia de un caso clínico de un hombre de 41 años, procedente de Bolivia, antecedentes de colecistectomía y apendicectomía. En 2013 presenta ingesta cáustica por intento de suicidio con necrosis total de esófago y estómago lo cual requirió en su país de origen, una esófago gastrectomía proximal de urgencia con ligadura a nivel del esfinter cricofaríngeo, pleurostomías y yeyunostomía de alimentación. Luego de 2 meses de hospitalización, logra ser dado de alta con alimentación por yeyunostomía. En los próximos 3 años presentó una calidad de vida muy deteriorada con aporte nutricional deficiente, con manejo de apoyo psiquiátrico (puntaje GIQLI = 73) con afagia, tos frecuente y salivando permanentemente, sin posibilidad de manejo quirúrgico en su país para revertir dicha situación por lo que es derivado a nuestro equipo.

Consulta en Chile el año 2016 para posible reconstitución de tránsito. Es evaluado, destacando un paciente en afagia durante los últimos 3 años, alimentándose por yeyunostomía, enflaquecido (IMC $=18,9)$, con desnutrición calórica proteica severa (albúmina $=2,9$ ) y sarcopenia, con un examen físico cardiopulmonar normal. El paciente se preparó con nutrición parenteral y enteral intensiva con aportes vitamínicos, oligoelementos mejorando así sus niveles proteicos, dando el equipo de nutrición el pase para su operación.
Se inicia el estudio ambulatorio con:

- Radiografía esófago-estómago-duodeno la cual muestra ausencia de paso de contraste distal a los senos piriformes (Figura 1).

- Endoscopia digestiva alta que confirma hallazgos radiológicos (Figura 2).

- Enema baritada y colonoscopia: sin hallazgos patológicos.

- Tomografía computada (TC) de tórax, abdomen y pelvis: sin hallazgos patológicos.

- Paralelamente, se analizó conjuntamente al equipo de microcirugía, con la finalidad de potenciar la irrigación del tubo digestivo interpuesto con cirugía microvascular cervical, por lo que se solicita un Angio TC de cuello que informa vasos cervicales sin hallazgos de carácter patológico.

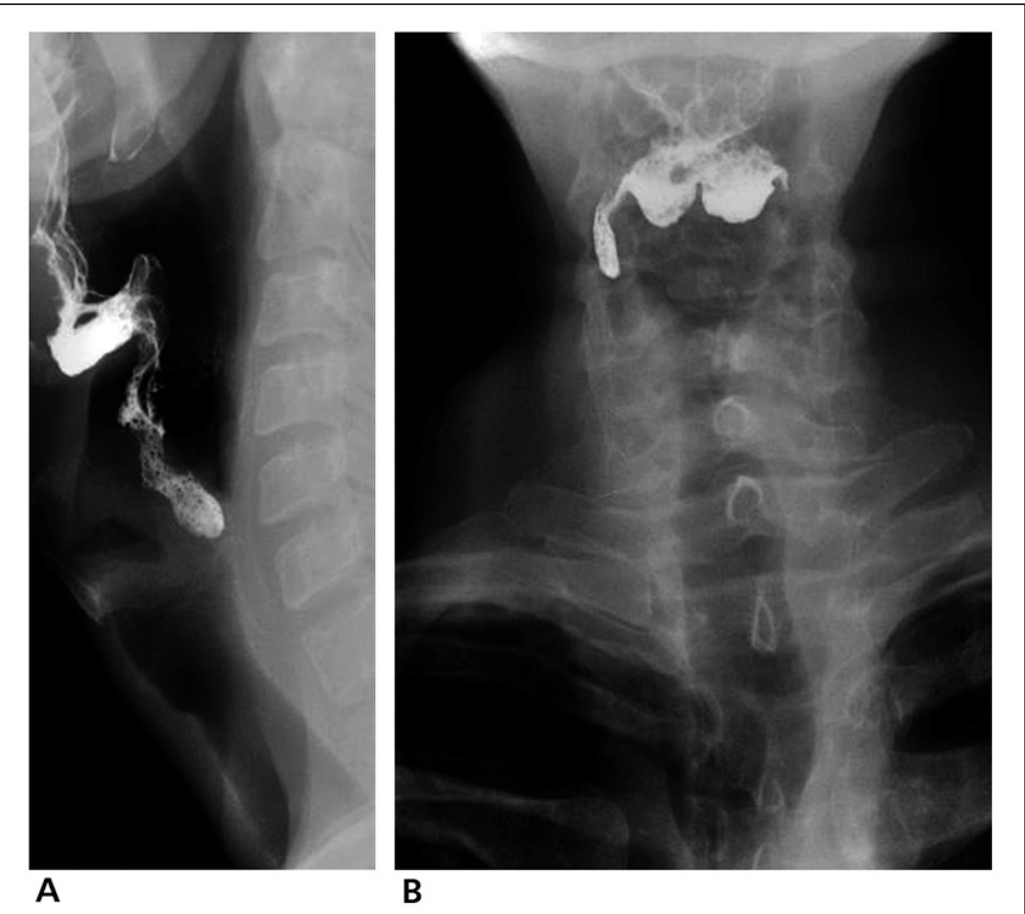

Figura 1. Radiografía baritada orofaríngea: estenosis total a nivel del seno piriforme. Visión lateral (A) y frontal (B). Sin paso de contraste a esófago.

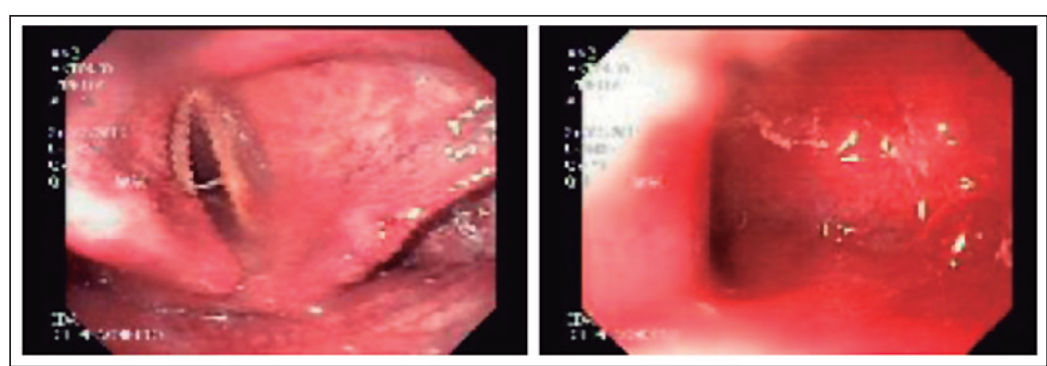

Figura 2. Endoscopia preoperatoria: estenosis total a nivel cricifaríngeo sin paso a distal. 


\section{CASOS CLÍNICOS}

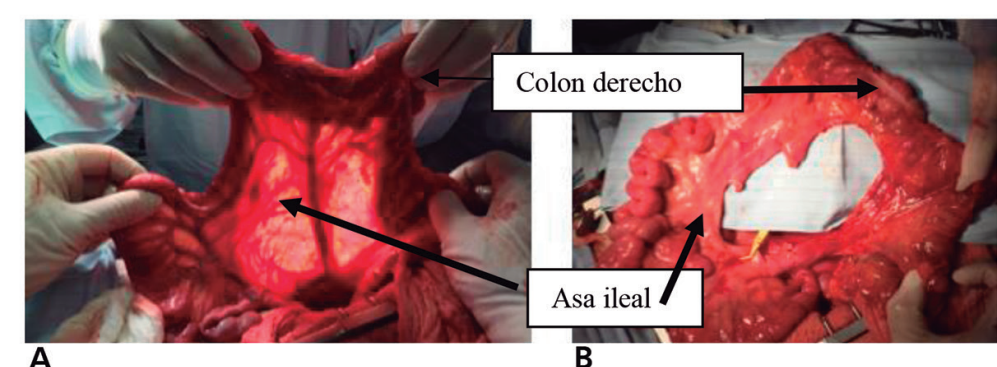

A

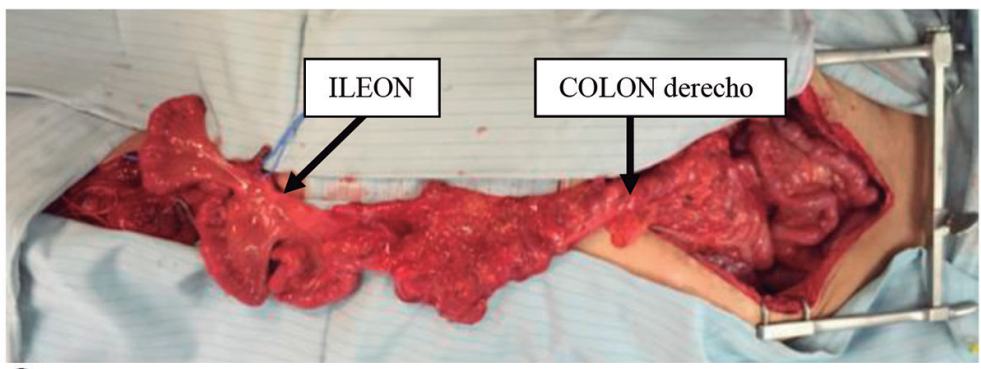

C
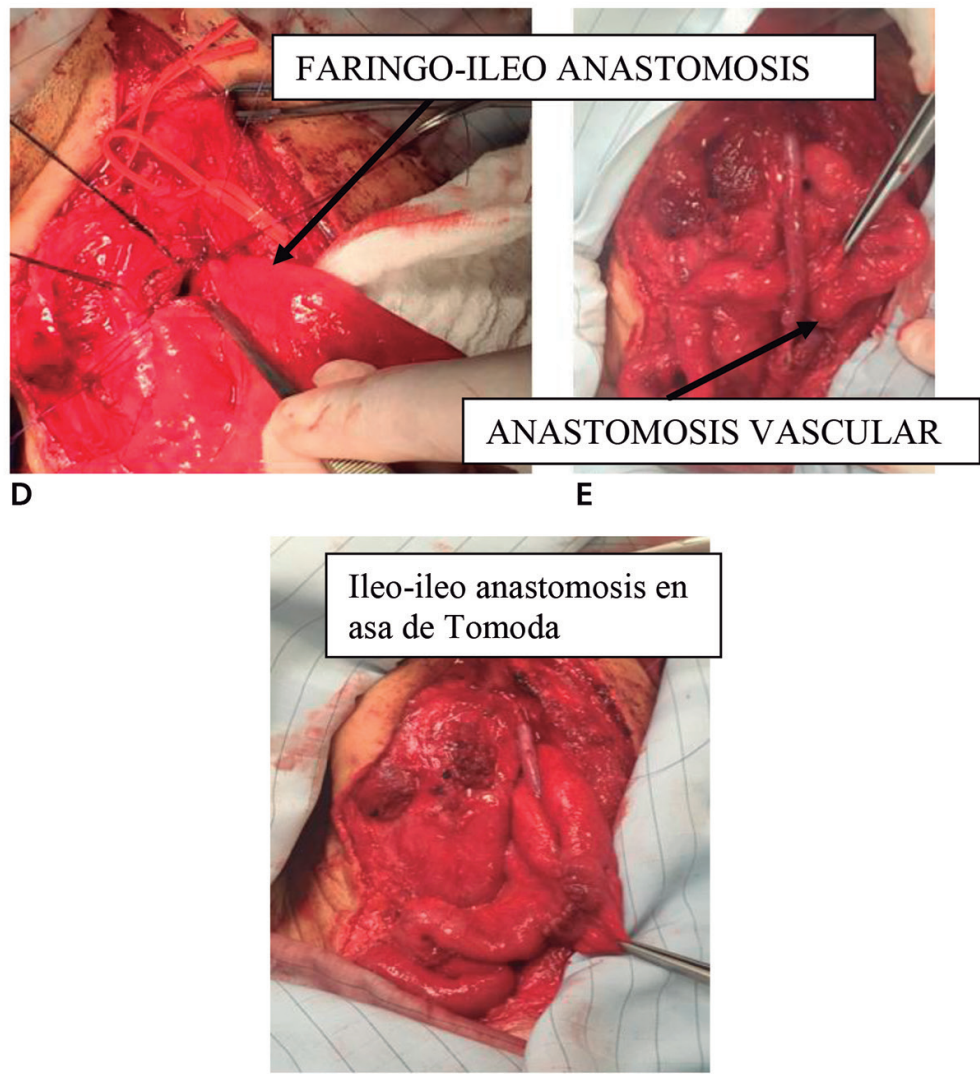

F

Figura 3. Imágenes intraoperatorias. Tiempo abdominal. A) Transiluminación de vaso íleo-ceco-apendico-cólico. B) Disección de íleon distal con clamp (amarillo) en última arcada vascular. C) Presentación de tubo ileocólico isoperistáltico desde abdomen (derecha) a cuello (izquierda). Tiempo cervical. D) Faringo-íleo anastomosis. E) Anastomosis vascular desde con interposición de vena yugular externa. F) Visión final con anastomosis de asa de Tomoda.
Se plantea como única alternativa de tratamiento el efectuar una interposición de colon con faringoíleo-colo anastomosis y suplemento vascular microquirúrgico para asegurar la irrigación sanguínea. Se prepara el colon derecho preservando cólica media y derecha seccionando el tronco íleo-cecoapendico-cólico, se incluye un segmento de íleon distal con sus últimas arcadas vasculares arterial y venosa.

Considerando este procedimiento como primera opción terapéutica, se indica hospitalización para evaluación conjunta por equipos de nutrición médica intensiva y kinesioterapia, para así llevar al paciente a la intervención quirúrgica, en las mejores condiciones nutricionales (por medio de nutrición enteral con arginina por yeyunostomía y aporte de micronutrientes endovenoso ajustados a requerimientos calculados) y motoras (a través de kinesioterapia motora y respiratoria preoperatoria).

Previo a la intervención se preparó el colon con polietilenglicol.

\section{Técnica quirúrgica}

\section{Cirugía abdominal}

Decúbito supino antisepsia con clorhexidina, campos estériles. Laparotomía media supra e infraumbilical. Se evidencian adherencias laxas omentales, colon de aspecto sano y bien preparado. En íleon distal, a $10 \mathrm{~cm}$ de válvula ileocecal se aprecia divertículo de Meckel no complicado. Espacio supra-meso-cólico con múltiples adherencias omento-peritoneo-viscerales.

Se procede a liberación de omento mayor de colon transverso y ascendente. Decolamiento de colon derecho. Identificación de pedículos vasculares cólicos medios, derecho, íleo-cólicos y de íleon distal. Se preservan vasos cólicos medios y derecho, seccionando rama íleo-ceco-apendico-cólica en su origen, conservando ultimas arcadas vasculares de mesenterio íleal a emplear obteniendo un segmento de íleon terminal de $20 \mathrm{~cm}$. Se advierte existencia de un divertículo de Meckel practicándose diverticulectomía con stapler carga blanca $45 \mathrm{~mm}$ (Medtronic ${ }^{\circledR}$ ) que se envía a biopsia diferida. Sección de colon transverso a la izquierda de vasos cólicos medios con stapler carga lila $60 \mathrm{~mm}$ (Medtronic ${ }^{\circledR}$ ), sección de íleon distal a $20 \mathrm{~cm}$ de válvula ileocecal con stapler carga blanca $30 \mathrm{~mm}$ (Medtronic ${ }^{\circledR}$ ). Previo al ascenso se comprueba adecuado pulso de todas las arcadas vasculares del colgajo visceral (Figura 3).

Tunelización retroesternal roma. Se objetiva 
apertura de pleura derecha la cual logra cerrarse en extremo distal con Monocryl 3/0. Pleurostomía derecha intercostal (24F) fijada con seda.

Ascenso retroesternal de íleon distal y colon derecho iso-peristáltico hasta región cervical.

\section{Cirugía cervical}

Cervicotomía izquierda sobre cicatriz previa. Proceso cicatricial importante. Abordaje por borde anterior de músculo esternocleidomastoideo, sección de omohioideo, identificación de vasos tiroideos superiores y carótida externa. Disección de elementos vasculares del cuello por parte del equipo de microcirugía. Se identifica vena tiroidea inferior de buen calibre, arteria ausente. Pedículos vasculares preparados por equipo de microcirugía.

Al realizar posicionamiento de órgano ascendido, se aprecia falta de aproximadamente de $10 \mathrm{~cm}$ entre pedículo de colgajo y arteria tiroidea superior por lo que se reseca segmento de vena yugular externa para confección de puente venoso, el cual se realiza con sutura de Ethylon ${ }^{\circledR}$ 9/0 continuo a cabos arteriales proximal y distal, comprobándose buen flujo. Anastomosis venosa con Ethylon ${ }^{\circledR}$ 9/0 continuo a vena tiroidea inferior, se comprueba buen flujo. Se aprecia mejor perfusión y motilidad de asa intestinal al fin del procedimiento (Figura 4).

Después de anastomosis vasculares, se fija intestino delgado a opérculo torácico, se deja drenaje Penrose cervical, cierre de músculo esternocleidomastoideo izquierdo con Vicryl 3/0, cierre de platisma y subdérmico con Vicryl $3 / 0$ y piel con grapas metálicas.

Faringo-íleo-anastomosis latero lateral manual doble plano con Monocryl ${ }^{\circledR} 3 / 0$, tutorizada con sonda 36F. Anastomosis íleo-leal en asa de Tomoda a nivel cervical con Monocryl ${ }^{\circledR}$ 3/0 (Figura 5). Esta técnica se eligió para obtener una anastomosis faringoileal más amplia y mejor irrigada sin un extremo cerrado que pueda significar un problema posterior y logrando un mejor vaciamiento a distal por tener 2 vías de salida.

Se completa el procedimiento con duodeno-coloanastomosis latero lateral manual doble plano con Monocryl 3/0 e íleo-transverso anastomosis latero lateral mecánica con stapler blanco $60 \mathrm{~mm}$ (Medtronic $^{\circledR}$ ) y cierre de enterotomía con Monocryl ${ }^{\circledR} 3 / 0$. Cierre de brechas con seda $3 / 0$. Drenaje de latex fino a anastomosis duodenal exteriorizado por flanco derecho, fijado con seda. Laparorrafia con vicryl 1 continuo, celular subcutáneo con Vicryl ${ }^{\circledR} 3 / 0$ y piel con grapas metálicas.

Duración de la cirugía fue de 6 h 55 min. Sangrado total aproximado de $250 \mathrm{cc}$.
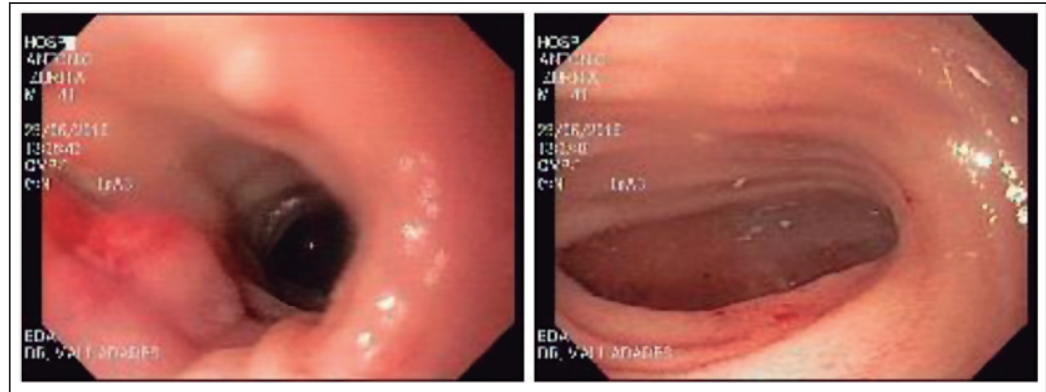

Figura 4. Endoscopia digestiva alta postoperatoria. Anastomosis e íleon posanastomótico sin signos de complicación.

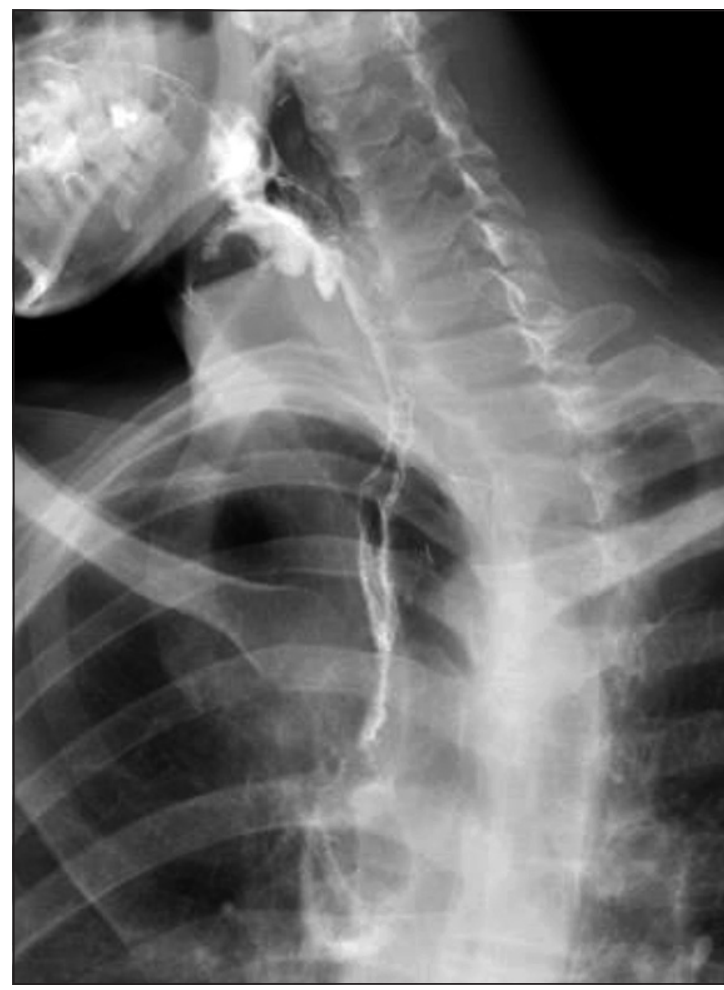

Figura 5. Esofagograma con bario posoperatorio y posdilatación endoscópica. Adecuado paso de contraste a interposición colo-íleal.

\section{Evolución postoperatoria}

Los primeros días es hospitalizado en unidad de paciente crítico para monitorización y evaluación multidisciplinaria estrecha.

En lo quirúrgico, se objetivó al 2 y $4^{\circ}$ día posoperatorio (DPO), 2 episodios de hemorragia digestiva alta (melena) de bajo volumen, sin repercusión hemodinámica ni caída de hemoglobina, las cuales fueron manejadas médicamente. En los controles radiológicos posoperatorios no se evidencia dilatación patológica de segmento de colon interpuesto. Dado 
estabilidad clínica, se traslada a sala de cuidados básicos, retira sonda Foley y drenaje cervical al $5^{\circ}$ DPO.

En lo nutricional, inicialmente recibe nutrición parenteral total complementaria a aporte de solución glucosada por yeyunostomía desde el $4^{\circ}$ DPO. Previa a la ingesta oral, se realiza un control de anastomosis con medio de contraste hidrosoluble oral, que muestra ausencia de paso de contraste a íleon distal, deteniéndose en senos piriformes de orofarinx. Se realiza endoscopia digestiva alta la que descarta estenosis anastomótica (Figura 4). Se mantiene con nutrición enteral complementaria por yeyunostomía.

Paralelamente, al inicio de ingesta oral de solución glucosada, se solicitó evaluación por equipo de fonoaudiología para rehabilitación de deglución.

Se retira pleurostomía derecha al $9^{\circ}$ día postoperatorio.

Se suspende nutrición parenteral al $15^{\circ} \mathrm{DPO}$, manteniendo nutrición oral y enteral, dado disfagia persistente se requirió dilatación endoscópica neumática hasta $20 \mathrm{~mm}$ con balón hidrostático, el control de anastomosis posterior muestra adecuado paso de contraste a distal (Figura 5), posteriormente se logra ingesta oral satisfactoria. El paciente es dado de alta al $17^{\circ} \mathrm{DPO}$ con nutrición oral y enteral por yeyunostomia complementaria.

Al mes posoperatorio, manteniendo rehabilitación fonoaudiológica, sin necesidad de nuevas dilataciones, logra aporte oral del $100 \%$ de sus requerimientos nutricionales y se retira yeyunostomía, indicándose suplemento vitamínico. Al $6^{\circ}$ mes posoperatorio cuenta con un control endoscópico que evidencia anastomosis amplia sin úlceras. Al año posoperatorio el paciente se encuentra alimentándose por vía oral en forma completa, se mantiene suplemento vitamínico obteniéndose un adecuado estado nutricional y su calidad de vida mejoró con una optimización del puntaje GIQLI preoperatorio de 73, a un puntaje postoperatorio de 122 .

El paciente ha sido controlado a los 18 meses de operado, está contento de su actual calidad de vida y otorgó el consentimiento para la publicación de su caso.

\section{Discusión}

Existen escenarios donde la reconstitución del tránsito digestivo alto con estómago no es posible de efectuar, ya sea por pacientes con gastrectomías previas, presencia de gastrostomías que imposibiliten su uso, pacientes con injuria gástrica secundaria a ingesta cáustica y/o en pacientes con ascenso gástri- co fallido por necrosis del tubo gástrico requiriendo un nuevo sustituto. En estos escenarios el ascenso de colon es la siguiente opción a escoger ${ }^{3,4,6}$. Existen ocasiones en las cuales ni siquiera se dispone de esófago cervical y la anastomosis se debe efectuar a nivel faríngeo ${ }^{4,7-9}$. El caso expuesto presenta un hombre sin posibilidades de reconstrucción gástrica, motivo por el cual se empleó un segmento de colon. En esta técnica se han descrito preferencias por distintos segmentos colónicos; quienes abogan por el colon izquierdo justifican una mayor constancia en su irrigación con menor riesgo de infarto o congestión y diámetros luminales menores, lo que facilitaría sus anastomosis ${ }^{7}$. El uso de colon transverso es más infrecuente, reservándose para situaciones con imposibilidad de emplear colon izquierdo o derecho, sin embargo, hay autores que la consideran de elección ${ }^{6-9}$

El uso tanto de colon izquierdo como transverso se caracterizan por la falta de mecanismo antireflujo de secreciones bilio-pancreáticas. En este contexto, emplear el colon derecho con un segmento de íleon distal (ileocoloplastía isoperistáltica), ofrece tener una válvula antirreflujo (válvula íleocecal), con una menor disección quirúrgica y un mejor diámetro anastomótico proporcionado por la concordancia del íleon distal y esófago cervical. Este argumento es el que justifica la preferencia de los autores, lo cual ha sido publicado previamente ${ }^{7}$. Una desventaja de este colgajo, es la inconstancia de sus arcadas vasculares. Hay estudios que han determinado una presencia de arteria cólica derecha en un $33,4 \%$ de los casos $^{9}$. Otros trabajos han estudiado la frecuencia de presentación de arteria marginal, siendo esta discontinua entre el 5\% y hasta $70 \%$ de los pacientes ${ }^{10-12}$. La decisión definitiva de qué segmento a emplear depende de la preferencia y experiencia de cada equipo quirúrgico ${ }^{9-15}$.

Considerando estos argumentos, para prevenir isquemia del segmento intestinal colo-íleal interpuesto, se planificó una potenciación vascular del colgajo con anastomosis microquirúrgica a vasos cervicales, similar a los injertos yeyunales libres.

Una forma de planificar qué tipo de colgajo colónico realizar, es empleando un AngioTC 3-D de abdomen y pelvis, el cual posee un $97,1 \%$ de certeza diagnóstica anatómica del árbol vascular mesentérico y cólico, una sensibilidad de $85,7 \%$, especificidad del $95,2 \%$, un valor predictivo positivo y negativo de $94,7 \%$ y $97,5 \%$ respectivamente ${ }^{16,17}$.

Independiente del segmento de colon a utilizar se reporta alta tasa de morbilidad asociada a este tipo de procedimientos. Una revisión actualizada respecto a reemplazo esofágico, analizó 16 estudios 
realizados en los últimos 17 años, con más de 1.000 pacientes en total, la tasa de complicaciones reportadas fue de: necrosis del colgajo: 0-14\% (centros de alto volumen entre $0-2 \%$ ), fístulas anastomóticas: $0-50 \%$ (centro de alto volumen entre 0-12,6\%), estenosis anastomótica: 0-32\%, complicaciones respiratorias: $10-42 \%$, mortalidad quirúrgica: $0-16,7 \%$ (centros de alto volumen entre $0-7 \%)^{18-24}$.

Últimamente, para asegurar una adecuada irrigación sanguínea del órgano ascendido, varios autores han reportado casos o series pequeñas de pacientes utilizando anastomosis microquirúrgicas entre los vasos del meso íleal y troncos vasculares del cuello con excelentes resultados. Saeki reportó 21 pacientes sometidos a interposición de colon utilizando el hemicolon derecho a través de la vía subcutánea y realizar una anastomosis venosa microvascular para todos los pacientes y sólo realizar una anastomosis arterial microvascular cuando el flujo sanguíneo arterial era insuficiente. La neumonía se produjo en cuatro pacientes $(19,0 \%)$, se observó fuga anastomótica en cinco pacientes $(23,8 \%)$, sin embargo, no se observó necrosis de colon. Uchiyama y otros han presentado resultados aún mejores con este procedimiento $^{25-29}$.

En el caso presentado justamente se tomó en cuenta estas experiencias para plantear el manejo de esta desafiante situación quirúrgica.

Respecto a la resección parcial del manubrio esternal y cabeza clavicular izquierda en ascenso retroesternal propuesta por algunos autores ${ }^{6}$, los autores del presente artículo no lo estiman necesario en caso de usar el colon derecho como colgajo, dado que una adecuada disección del opérculo torácico y el menor volumen del íleon distal, hacen de esta resección un elemento innecesario y mórbido. Sí es importante, bajo nuestra apreciación, fijar adecuadamente el colgajo colo-íleal a la fascia prevertebral y músculos infrahioideos, con la finalidad de evitar tensión en la anastomosis proximal y conjuntamente ocluir de forma eficaz el opérculo torácico, de tal manera que si existiera una fuga anastomótica el fluido purulento no escurra hacia el mediastino.

En Chile, hay pocos reportes referentes a este tema puntual ${ }^{4,7,14,15,30-32}$.

Como conclusión, creemos que estos casos representan una situación clínica muy desafiante, que debe ser evaluada y manejada de manera multidisciplinaria con la participación de cirujanos gastrointestinales y microvasculares, nutriólogos, anestesiólogos, fonoaudiólogos, kinesiterapeutas, endoscopistas y apoyo psiquiátrico para restablecer una calidad de vida normal.

La adecuada preparación nutricional es básica para obtener una buena evolución postoperatoria desde el punto de vista local y general.

\section{Responsabilidades éticas}

Protección de personas y animales. Los autores declaran que para esta investigación no se han realizado experimentos en seres humanos ni en animales.

Confidencialidad de los datos. Los autores declaran que en este artículo no aparecen datos de pacientes.

Derecho a la privacidad y consentimiento informado. Los autores declaran que en este artículo no aparecen datos de pacientes.

\section{Financiación}

Los autores declaran no haber recibido ninguna financiación para la realización de este trabajo.

\section{Conflicto de intereses}

Los autores declaran no tener ningún conflicto de intereses.

\section{Bibliografía}

1. Wormuth JK, Heitmiller RF. Esophageal conduit necrosis. Thorac Surg Clin. 2006; 16:11-22.

2. Marks JL, Hofstetter WL. Esophageal reconstruction with alternative conduits. Surg Clin North Am. 2012;92:1287-97.

3. Belsey R. Reconstruction of the esophagus with left colon. Thorac Cardiovasc Surg. 1965;49:33.
4. Braghetto I, Cardemil G, Csendes A, Venturelli A, Herrera M, Korn O, et al. Digestive tract reconstitution after failed esophago-gastro or esophagocoloanastomosis. ABCD Arq Bras Cir Dig. 2013;26:7-12.

5. Barkley $\mathrm{C}$, Orringer MB, Iannettoni MD, Yee J. Challenges in reversing esophageal discontinuity operations. Ann Thorac Surg. 2003;76:989-94.

6. Maish MS, DeMeester SR. Indications and technique of colon and jejunal interpositions for esophageal disease. Surg Clin North Am. 2005;85:505-14.

7. Braghetto I, Csendes A, Amat J, Parada M, Schutte H, Lizana C, et al. Reconstrucción del tránsito esofágico con interposición de colon. Rev Chil Cir. 1994;46:477-84.

8. Weiss AR, Hackl C, Soeder Y, Schlitt HJ, Dahlke MH. Ileo-right hemi-colonic cervical pull-up on a non-supercharged 
ileocolic arterial pedicle: A technical and case report. World J Gastroenterol. 2016;22:3869-74.

9. Dickinson KJ, Blackmon SH. Management of Conduit Necrosis Following Esophagectomy. Thorac Surg Clin. 2015;25:461-70.

10. DeMeester SR. Colon interposition following esophagectomy. Dis Esophagus. 2001;14:169-72.

11. Murono K, Kawai K, Ishihara S, Otani K, Yasuda K, Nishikawa T, et al. Evaluation of the vascular anatomy of the right-sided colon using three-dimensional computed tomography angiography: a singlecenter study of 536 patients and a review of the literature. Int J Colorectal Dis. 2016;31:1633-8.

12. Briel JW, Tamhankar AP, Hagen JA, DeMeester SR, Johansson J, Choustoulakis E, et al. Prevalence and risk factors for ischemia, leak, and stricture of esophageal anastomosis: gastric pullup versus colon interposition. J Am Coll Surg. 2004;198:536-41.

13. Knezević JD, Radovanović NS, Simić AP, Kotarac MM, Skrobić OM, Konstantinović VD, et al. Colon interposition in the treatment of esophageal caustic strictures: 40 years of experience. Dis Esophagus 2007;20:5304.

14. Abularach R, Venturelli F, Cerda R, Urizar A, Lira E, Haito Y, et al. Interposición de colon transverso como alternativa de reconstrucción tras la esofagogastrectomía total. Rev Chil Cir. 2011;63:432-6.

15. Braghetto I, Cardemil G, Csendes A, Domínguez C, Musleh M, Korn O, et al. Reconstitución de tránsito esofágico con interposición de colon derecho. Rev Chil Cir. 2015;67:271-7.
16. Nesgaard JM, Stimec BV, Bakka AO, Edwin B, Ignjatovic D. RCC study group. Navigating the mesentery: a comparative pre- and per-operative visualization of the vascular anatomy. Colorectal Dis. 2015; 17:810-8.

17. Nesgaard JM, Stimec BV, Bakka AO, Edwin B, Ignjatovic D. RCC study group Navigating the mesentery: part II. Vascular abnormalities and a review of the literature. Colorectal Dis. 2017;19:656666.

18. Bakshi A, Sugarbaker D, Burt B. Alternative conduits for esophageal replacement. Ann Cardiothorac Surg. 2017;6:137-43.

19. De Delva PE, Morse CR, Austen WG Jr, Gaissert HA, Lanuti M, Wain JC, et al. Surgical management of failed colon interposition. Eur J Cardiothorac Surg. 2008;34:432-7; discussion 437.

20. Okazaki M, Asato H, Takushima A, Nakatsuka T, Ueda K, Harii K. Secondary reconstruction of failed esophageal reconstruction. Ann Plast Surg. 2005;54:530-7.

21. Theile DE, Robinson DW. Microvascular salvage for failed colonic interposition. Aust N Z J Surg. 1987;57:749-52.

22. Coleman JJ 3rd. Reconstruction of the pharynx and cervical esophagus. Semin Surg Oncol. 1995;11:208-20.

23. Cassivi SD. Leaks, strictures and necrosis: a review of anastomotic complications following esophagectomy. Seminar in Thoracic and Cardiovasc. Surgery. 2004;16:124-32.

24. Barkley C, Orringer MB, Iannettoni MD, Yee J. Challenges in reversing esophageal discontinuity operations. Ann Thorac Surg. 2003;76:989-94.
25. Saeki H, Morita M, Harada N, Egashira A, Oki E, Uchiyama H, et al. Esophageal replacement by colon interposition with microvascular surgery for patients with thoracic esophageal cancer: the utility of superdrainage. Dis Esophagus. 2013;26:50-6.

26. Uchiyama H, Morita M, Toh Y, Saeki H, Kakeji Y, Matsuura H, et al. Superdrainage of the ileocolic vein to the internal jugular vein interposed by an inferior mesenteric vein graft in replacing the esophagus with the right hemicolon. Surg Today. 2010;40:578-82.

27. Kesler KA, Pillai ST, Birdas TJ, Rieger KM, Okereke IC, Ceppa D, et al. "Supercharged" isoperistaltic colon interposition for long-segment esophageal reconstruction. Ann Thorac Surg. 2013;95:1162-8.

28. Blackmon SH, Correa AM, Skoracki R, Chevray PM, Kim MP, Mehran RJ, et al. Supercharged pedicled jejunal interposition for esophageal replacement: a 10-year experience. Ann Thorac Surg. 2012;94:1104-11.

29. Swisher SG, Hofstetter WL, Miller MJ. The supercharged microvascular jejunal interposition. Semin Thorac Cardiovasc Surg. 2007;19:56-65.

30. De Carolis V, Braghetto I, Cardemil G. Reconstrucción faringo-esofágica cervical con colgajo libre de yeyuno microvascularizado. Rev Chil Cir. 1992;44: 105-8.

31. Burmeister R, Benavides C, García C, Reyes C. Reemplazo esofágico con colon. Rev Chil Cir. 1997;49:76-81.

32. Lois J, Viterbo A, Gaete P. Reemplazo de esófago por colon en la estenosis cáustica. Arch Soc Cir Chile 1971;23: 26-9. 